Check for updates

Cite this: Nanoscale Adv., 2019, 1, 953

Received 17th September 2018 Accepted 5th November 2018

\title{
Effects of precursor pre-treatment on the vapor deposition of $\mathrm{WS}_{2}$ monolayers $\uparrow$
}

\author{
Mei Er Pam, ${ }^{\text {ab }}$ Yumeng Shi, ${ }^{\text {acd }}$ Junping $\mathrm{Hu}^{\mathrm{a}}$ Xiaoxu Zhao, (D) Jiadong Dan, \\ Xue Gong, ${ }^{\text {ac }}$ Shaozhuan Huang, ${ }^{a}$ Dechao Geng, ${ }^{a}$ Stephen Pennycook, ${ }^{\text {fg }}$ \\ Lay Kee Ang ${ }^{\text {ab }}$ and Hui Ying Yang (iD *a
}

DOI: $10.1039 / c 8 n a 00212 f$

rsc.li/nanoscale-advances

Transition metal oxide powders have been widely used as the growth precursors for monolayer transition metal dichalcogenides (TMDCs) in chemical vapor deposition (CVD). It has been proposed that metal oxide precursors in the gas phase undergo a two-step reaction during CVD growth, where transition metal sub-oxides are likely formed first and then the sulfurization of these sub-oxides leads to the formation of TMDCs. However, the effects of stoichiometry of transition metal oxide precursors on the growth of TMDC monolayers have not been studied yet. In this contribution, we report the critical role of the $\mathrm{WO}_{3}$ precursor pre-annealing process on the growth of $\mathrm{WS}_{2}$ monolayers. Besides, several $\mathrm{WO}_{3}$ precursors with different types of oxygen vacancies have also been prepared and investigated by $\mathrm{X}$-ray powder diffraction (XRD), X-ray photoelectron spectroscopy (XPS) and density functional theory calculation. Among all the non-stoichiometric $\mathrm{WO}_{3}$ precursors, thermally annealed $\mathrm{WO}_{3}$ powder exhibits the highest oxygen vacancy concentration and produces $\mathrm{WS}_{2}$ monolayers with significantly improved quality in terms of lateral size, density, and crystallinity. Our comprehensive study suggests that the chemical composition of transition metal oxide precursors would be

${ }^{a}$ Pillar of Engineering Product Development, Singapore University of Technology and Design, 8 Somapah Road, Singapore 487372, Singapore. E-mail: yanghuiying@sutd. edu.sg

${ }^{b}$ Science and Math Cluster, Singapore University of Technology and Design, 8 Somapah Road, Singapore 487372, Singapore

'International Collaborative Laboratory of 2D Materials for Optoelectronic Science \& Technology of Ministry of Education, Engineering Technology Research Center for $2 D$ Material Information Function Devices and Systems of Guangdong Province, College of Optoelectronic Engineering, Shenzhen University, Shenzhen 518060, China ${ }^{d}$ Engineering Technology Research Center for $2 D$ Material Information Function Devices and Systems of Guangdong Province, College of Optoelectronic Engineering, Shenzhen University, Shenzhen 518060, China

${ }^{e}$ Department of Chemistry, National University of Singapore, 3 Science Drive 3, Singapore 117543, Singapore

${ }^{f}$ Department of Materials Science and Engineering, National University of Singapore, 9 Engineering Drive 1, Singapore 117575, Singapore

${ }^{g}$ NUS Graduate School for Integrative Sciences and Engineering, National University of Singapore, 13 Centre for Life Science, \#05-01, 28 Medical Drive, Singapore 117456, Singapore

† Electronic supplementary information (ESI) available. See DOI: 10.1039/c8na00212f fundamentally critical for the growth of large-area and high-quality $W_{2}$ monolayers, which further pave the way for revealing their intrinsic properties and unique applications.

\section{Introduction}

The extraordinary electrical, optical, thermal and mechanical properties, which exist in monolayer transition metal dichalcogenides (TMDC) materials, offer various promising applications in future ultrathin logic devices, nanogenerators, flexible electronics and optoelectronic devices. ${ }^{\mathbf{1 - 4}}$ Specifically, the tunable properties of band offset, carrier density, ${ }^{5}$ and band gap $^{6-8}$ make TMDCs one of the most extensively researched subjects for practical and fundamental studies. Monolayer tungsten disulfide $\left(\mathrm{WS}_{2}\right)$ has been intensively studied as a next generation nanoelectronic and optoelectronic material due to its indirect to direct band gap tunability, ${ }^{\mathbf{9}, 10}$ high on/off current ratio, ${ }^{\mathbf{1 1}, 12}$ high thermal stability, ${ }^{13}$ excellent electrostatic integrity $^{14}$ and strong photoluminescence. ${ }^{15,16}$ Additionally, $\mathrm{WS}_{2}$ monolayers have shown an interesting spin orbit coupling effect resulting from the breaking of inversion symmetry of their crystal lattices, and the strong magnetic field interaction in $\mathrm{WS}_{2}$ monolayers favors their applications in optoelectronic and spintronic devices. ${ }^{17,18}$

Various approaches have been employed to prepare $\mathrm{WS}_{2}$ monolayers. The layered structure of $\mathrm{WS}_{2}$ allows the mechanical or chemical exfoliation of monolayer crystals from their bulk counterparts. However, the exfoliated flakes show random thickness, size and shape that create great difficulties in large scale industrial applications. ${ }^{\mathbf{1 6}}$ Chemical vapor deposition (CVD) has been widely adapted as one of the most effective strategies to grow wafer-scale two-dimensional (2D) materials including graphene, boron nitride, and TMDCs. ${ }^{19-23}$ In the pioneering studies of monolayer $\mathrm{WS}_{2}$ synthesis, tungsten trioxide $\left(\mathrm{WO}_{3}\right)$ powder was chosen as the growth precursor and a low concentration of hydrogen $\left(\mathrm{H}_{2}\right)$ gas was usually introduced to reduce $\mathrm{WO}_{3}$ to tungsten suboxide. ${ }^{22,24,25}$ Nevertheless, excessive hydrogen supply could also have an etching effect and 
induce various structural defects in monolayer $\mathrm{WS}_{2} \cdot{ }^{\mathbf{1 6 , 2 5 , 2 6}}$ Traditionally, tungsten oxides have been intensively studied previously for photocatalysis, ${ }^{27,28}$ electrochemistry, ${ }^{29}$ electrochromic application, ${ }^{\mathbf{3 0 , 3 1}}$ etc. According to the literature, $\mathrm{WO}_{3}$ powder is usually found in a non-stoichiometric composition and the $\mathrm{W}$ element may show various chemical states due to the spontaneous reduction of $\mathrm{WO}_{3}$ under ambient conditions. ${ }^{30}$ It has been demonstrated that the sulfurization process of transition-metal suboxides in the CVD process plays a critical role in the nucleation and crystallization of TMDC monolayers. ${ }^{22,32}$

In the CVD process, the generation and evolution of transition metal sub-oxide species can significantly affect the morphology and edge structure of TMDCs, which lead to dramatically different optical, electronical and magnetic properties. $^{20}$ However, the study of the effects of chemical composition of $\mathrm{WO}_{3}$ on the CVD growth of $\mathrm{WS}_{2}$, in terms of quality, morphology and optical properties, is still lacking. Herein, we prepared various tungsten oxide precursors with different oxygen vacancy concentrations, that is, commercially available fresh $\mathrm{WO}_{3}\left(\mathrm{FWO}_{3}\right)$, thermally annealed $\mathrm{WO}_{3}\left(\mathrm{AWO}_{3}\right)$, air plasma treated $\mathrm{WO}_{3}\left(\mathrm{PTWO}_{3}\right)$ and thermally annealed $\mathrm{PTWO}_{3}$ powder $\left(\mathrm{PTAWO}_{3}\right)$ to provide tungsten suboxide sources. The results reveal that $\mathrm{AWO}_{3}$ powder with the highest oxygen vacancy content produces $\mathrm{WS}_{2}$ monolayers with significantly improved domain size, higher coverage density, and higher crystallinity. Besides, we further demonstrate that the use of $\mathrm{AWO}_{3}$ powder instead of the introduction of hydrogen gas during the growth of monolayer $\mathrm{WS}_{2}$ to provide a tungsten suboxide source will enhance the growth of monolayer $\mathrm{WS}_{2}$ and produce monolayer $\mathrm{WS}_{2}$ with suppressed atomic defects. The control synthesis of $\mathrm{WS}_{2}$ monolayers by precursor pretreatment provides further insights into the growth mechanism and enables the production of $\mathrm{WS}_{2}$ monolayers with high quality for the development of a broad range of optoelectronic and electronic applications.

\section{Experimental methods}

\section{Preparation of tungsten trioxide $\left(\mathrm{WO}_{3}\right)$ precursors}

The $\mathrm{WO}_{3}$ precursors were pretreated with heat, air plasma or both. Four $\mathrm{WO}_{3}$ precursors prepared with different methods were studied for comparison, including commercially available $\mathrm{WO}_{3}\left(\mathrm{FWO}_{3}\right)$ (Sigma-Aldrich), thermally annealed $\mathrm{WO}_{3}\left(\mathrm{AWO}_{3}\right)$, air plasma treated $\mathrm{WO}_{3}\left(\mathrm{PTWO}_{3}\right)$ and plasma treated annealed $\mathrm{WO}_{3}\left(\mathrm{PTAWO}_{3}\right)$ powder.

For $\mathrm{AWO}_{3}$ powder, $500 \mathrm{mg}$ of $\mathrm{WO}_{3}$ powder was loaded in an alumina boat, placed at the center of a furnace, and heated up to $500{ }^{\circ} \mathrm{C}$ in air for 30 minutes.

For $\mathrm{PTWO}_{3}$ powder, $500 \mathrm{mg}$ of $\mathrm{WO}_{3}$ powder was loaded in an alumina boat, placed at the center of a plasma reactor center (Plasma Cleaner PDC-32G-2). The sample was directly exposed to a direct inductively coupled plasma. During the plasma treatment process, the chamber was evacuated to 1.5 mTorr with a mechanical pump and the radio frequency power was turned to $\sim 18 \mathrm{~W}$, and at the same time air was introduced into the chamber and the process was continued for 5 minutes.

For $\mathrm{PTAWO}_{3}$ powder, $500 \mathrm{mg}$ of $\mathrm{WO}_{3}$ powder was first treated using air plasma with the same preparation steps as the plasma treated $\mathrm{WO}_{3}$ powder, and the powder was then annealed using the same procedure that we employed to prepare the annealed $\mathrm{WO}_{3}$ powder sample.

\section{Growth process of monolayer $\mathrm{WS}_{2}$}

The employed CVD process for monolayer $\mathrm{WS}_{2}$ is based on our previous report. ${ }^{33,34}$ Prior to the growth process, single-side polished sapphire substrates were washed with the solution mixture of $\mathrm{H}_{2} \mathrm{SO}_{4}$ and $\mathrm{H}_{2} \mathrm{O}_{2}$ and blow-dried with nitrogen gas. An alumina boat filled with $500 \mathrm{mg}$ pre-prepared $\mathrm{WO}_{3}$ powder (Sigma-Aldrich, 99.9\%) was located at the center of a furnace, and the cleaned sapphire substrates were placed facing down on the boat with $\mathrm{WO}_{3}$ powder as illustrated in Fig. S1. $\dagger$ A separate boat with sulfur powder (Sigma-Aldrich, 99.5\%) was located at the upstream of the furnace, which was heated up to the sulfur evaporation temperature at $120^{\circ} \mathrm{C}$ with a heating belt during the growth of monolayer $\mathrm{WS}_{2}$. The CVD chamber was initially pumped down to a base pressure of $\sim 1$ mTorr for 5 minutes and maintained at 15 torr with a $100 \mathrm{sccm}$ flow of argon gas during the synthesis process. The furnace was then heated from room temperature to $200{ }^{\circ} \mathrm{C}$ and the temperature was kept constant for 10 minutes to evaporate the water vapor in the quartz tube. The temperature was then further heated up to $950{ }^{\circ} \mathrm{C}$ at a ramp rate of $25^{\circ} \mathrm{C}$ per min and kept constant for 10 minutes. Finally, the growth chamber was cooled down to room temperature naturally. For the hydrogenated environmentally synthesized $\mathrm{WS}_{2}\left(\mathrm{HWS}_{2}\right)$ sample, the growth process was the same as that described above with commercially available $\mathrm{WO}_{3}$ powder except that $5 \%$ hydrogen gas is introduced into the tube furnace during the growth process.

\section{DFT calculation of the $\mathrm{WO}_{3}$ crystal structure}

DFT calculations were performed on the crystal structure of $\mathrm{WO}_{3}$ with and without oxygen vacancies. Our calculations were based on density functional theory (DFT) using plane-wave pseudopotentials with the exchange-correlation of PerdewBurke-Ernzerhof $(\mathrm{PBE})^{\mathbf{3 5 , 3 6}}$ form as implemented in the Vienna $A b$ initio Simulation Package (VASP). ${ }^{37}$ A cutoff energy of $500 \mathrm{eV}$ was employed for the plane wave expansion of the wave functions. The Brillouin zone was sampled with a $9 \times 5 \times 5$ Monkhorst-Pack $k$-point mesh ${ }^{38}$ for the structural optimization. The convergence criteria for the total energy and ionic forces were set to $10^{-5} \mathrm{eV}$ and $0.005 \mathrm{eV} \AA^{-1}$ respectively. Spin polarization was carefully tested and no spin-polarization effect was observed in our calculations.

\section{Characterization}

Photoluminescence (PL) and Raman measurements were obtained with a Witec Alpha300 Confocal Raman system with a laser excitation wavelength of $532 \mathrm{~nm}$ and grating density of 600 and $1800 \mathrm{~mm}^{-1}$ respectively. X-ray photoelectron spectroscopy (XPS, PHI Quantera II) with a monochromatic Al Ka source $(1486.69 \mathrm{eV})$, operated at a base pressure of $10^{-7} \mathrm{~Pa}$, was employed to obtain the chemical configuration spectra. X-ray diffraction (XRD, Bruker D8) with a $\mathrm{Cu} \mathrm{K} \alpha$ source was employed to identify the crystalline phases and structures of the prepared 
$\mathrm{WO}_{3}$ powders. The annular dark field scanning transmission electron microscope (ADF-STEM) image was captured on an aberration-corrected JEOL ARM-200F equipped with a cold field emission gun, operating at $80 \mathrm{kV}$. The convergence semi-angle of the probe is $\sim 30 \mathrm{mrd}$. STEM-ADF images were captured for a half angle range from $\sim 85$ to $280 \mathrm{mrd}$. A dwell time of $19 \mu \mathrm{s}$ per pixel was set for a single-scan image.

\section{Transfer}

The as-grown monolayer $\mathrm{WS}_{2}$ on sapphire was first spin coated with a thin layer of poly(methyl methacrylate) (PMMA) for one minute at a spin speed of $2000 \mathrm{rpm}$. Then, the edges of the sapphire substrate were removed and the substrate was immersed slowly in a hot solution of $3 \mathrm{~mol} \mathrm{~L}^{-1} \mathrm{NaOH}\left(160^{\circ} \mathrm{C}\right)$ to detach the PMMA layer from the sapphire substrate. The resulting sample was then delaminated in deionized water and washed a few times in deionized (DI) water. Finally, the sample was transferred onto a target substrate and carefully blow-dried with nitrogen gas. The PMMA coating was finally removed by using hot acetone vapor at $120{ }^{\circ} \mathrm{C}$ for 15 minutes. The samples for STEM study were prepared onto 200 mesh Quantifoil R2/1 micromachined copper grids from SPI Supplies, Structure Probe, Inc. using the same transfer method.

\section{Results and discussion}

Generally, the synthesis of 2D TMDCs with the chemical vapor deposition method is based on the direct competition and multiple elementary processes in two reaction pathways between the transition metal suboxide and sulfur. The reaction pathways consist of: (1) transition metal suboxide diffuses and adsorbs on the substrate, and then reacts with $\mathrm{S}$ to form monolayer TMDC; (2) transition metal suboxide directly reacts with $\mathrm{S}$ in the gas phase to form a TMDC and the resulting $\mathrm{WS}_{2}$ clusters adsorb, nucleate and form on the substrate. ${ }^{22,32}$ Both of the two reaction routes strongly rely on the evaporation and stable delivery of the tungsten source. The metastable state tungsten suboxide can provide a much easier control over the supply of the tungsten source, and thus facilitate the growth process and enhance the growth of $\mathrm{WS}_{2}$ monolayers. Therefore, the formation of tungsten suboxide plays a critical role in the nucleation and crystallization of TMDC monolayers. In a previous report, in order to promote the growth of monolayer $\mathrm{WS}_{2}$, hydrogen gas was introduced during the synthesis, which assists the reduction of tungsten trioxide. ${ }^{16,24,25}$ Fig. 1a illustrates the synthesis process of monolayer $\mathrm{WS}_{2}$ in an $\mathrm{Ar} / \mathrm{H}_{2}$ atmosphere in which hydrogen gas acts as a tungsten trioxide reductant to reduce tungsten oxide to form volatile tungsten suboxides $\left(\mathrm{WO}_{3}+x \mathrm{H}_{2} \rightarrow \mathrm{WO}_{3-x}+x \mathrm{H}_{2} \mathrm{O}\right){ }^{16,25,39}$ The resulting $\mathrm{WO}_{3-x}$ are readily volatile, easily evaporate and release the tungsten source. ${ }^{\mathbf{4 0 - 4 2}}$ The rapid evaporation of the tungsten source will eventually lead to an increase of nucleation sites and hence promote the growth of $\mathrm{WS}_{2}{ }^{24,25}$ Unfortunately, the excessive supply of hydrogen has etching effects on $\mathrm{WS}_{2}\left(\mathrm{WS}_{2}+\right.$ $\left.2 \mathrm{H}_{2} \rightarrow \mathrm{W}+2 \mathrm{H}_{2} \mathrm{~S}\right) \cdot{ }^{16,25}$ The excess hydrogen in the growth atmosphere will react with the as-grown $\mathrm{WS}_{2}$ flakes and remove the sulfur elements, resulting in sulfur vacancies. Precursor pretreatments such as thermal annealing and plasma treatment create oxygen vacancies in $\mathrm{WO}_{3}$, resulting in $\mathrm{WO}_{3-x}$ (see Fig. 1b). The formation of volatile $\mathrm{WO}_{3-x}$ suggests great potential in promoting the growth of monolayer $\mathrm{WS}_{2}$. Herein, to reveal the effects of oxygen vacancies in $\mathrm{WO}_{3}$ on the growth of $\mathrm{WS}_{2}$ monolayers, various tungsten trioxide powders with different oxygen vacancies were prepared, including thermally annealed $\mathrm{WO}_{3}\left(\mathrm{AWO}_{3}\right)$ powder, air plasma treated $\mathrm{WO}_{3}$ $\left(\mathrm{PTWO}_{3}\right)$ powder, air plasma treated and annealed $\mathrm{WO}_{3}$ $\left(\mathrm{PTAWO}_{3}\right)$ powder, and commercially available $\mathrm{WO}_{3}$ powder $\left(\mathrm{FWO}_{3}\right)$. We first employed $\mathrm{AWO}_{3}$ powder to obtain the optimized $\mathrm{WS}_{2}$ growth temperature under the same pressure of 15 torr (see Fig. $\mathrm{S} 1 \dagger$ ). As illustrated in Fig. S1, $\dagger$ an optimized growth temperature of $950{ }^{\circ} \mathrm{C}$ was determined for $\mathrm{WS}_{2}$ monolayer synthesis.

We further compared the as-grown $\mathrm{WS}_{2}$ monolayers produced from the four $\mathrm{WO}_{3}$ sources with different pre-treatment methods. The growth of monolayer $\mathrm{WS}_{2}$ strongly depends on the growth conditions, and in order to exclusively probe the effects of $\mathrm{WO}_{3}$ precursors on the CVD synthesis, regardless of

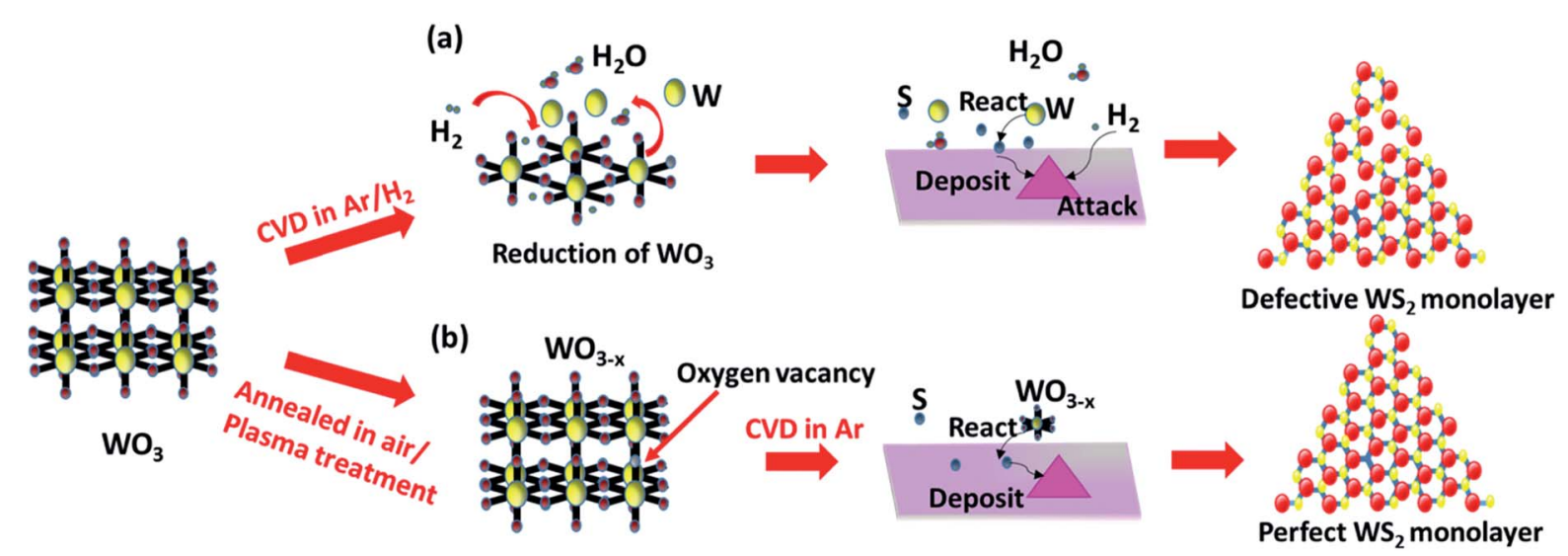

Fig. 1 Reaction mechanism for CVD growth of (a) the commercial precursor in an $\mathrm{Ar} / \mathrm{H}_{2}$ atmosphere; (b) pretreated precursors in an Ar only atmosphere. 
the precursor pre-treatment methods used, all the growth processes were carried out under the same growth conditions with a growth temperature of $950{ }^{\circ} \mathrm{C}$ and a growth time of 10 minutes. AFM analysis and Raman characterization (see Fig. S2 and $\mathrm{S} 3 \dagger$ ) were carried out to confirm the monolayer nature of the $\mathrm{WS}_{2}$. The AFM line profiles at the edge of all the samples demonstrate that the thicknesses are in the $0.6 \mathrm{~nm}$ to $0.9 \mathrm{~nm}$ range, which fall in the thickness range of monolayer $\mathrm{WS}_{2}$. The thickness value differences in such a small range are commonly observed due to the sample's surface conditions. We further compare their Raman peaks, and the samples show two characteristic peaks of monolayer $\mathrm{WS}_{2}$ : a sharp one at $355 \mathrm{~cm}^{-1}$ assigned to $E_{2 \mathrm{~g}}$ mode and a broad one at $355 \mathrm{~cm}^{-1}$ assigned to $A_{1 \mathrm{~g}}$ mode, matched well with previously reported Raman characterization of monolayer $\mathrm{WS}_{2} \cdot{ }^{15,16,43}$ Therefore, all the samples studied in our work are monolayer $\mathrm{WS}_{2}$, and changing the precursor treatment conditions has no effects on the sample's thickness. According to the statistical analysis over an area of $1000 \mu \mathrm{m} \times 750 \mu \mathrm{m}$ on the growth substrate (see Fig. S4 $\dagger$ ), the growth size of the monolayer $\mathrm{WS}_{2}$ is greatly influenced by the type of precursor. An average size of less than $10 \mu \mathrm{m}$ for $\mathrm{WS}_{2}$ monolayers was obtained with the $\mathrm{FWO}_{3}$ precursor, while $\mathrm{WS}_{2}$ monolayers with a maximum size of $\sim 70 \mu \mathrm{m}$ and an average size of $\sim 40 \mu \mathrm{m}$ could be achieved with the $\mathrm{AWO}_{3}$ precursor. $\mathrm{WS}_{2}$ monolayers with reduced density and an average lateral size of $\sim 10 \mu \mathrm{m}$ were obtained when the precursor was changed from $\mathrm{FWO}_{3}$ to $\mathrm{PTWO}_{3}$. Interestingly, a simple annealing process on the $\mathrm{PTWO}_{3}$ precursor resulted in $\mathrm{WS}_{2}$ monolayers with an average lateral size of $\sim 20 \mu \mathrm{m}$ and improved surface coverage on the growth substrate. Besides, as shown in the optical images in Fig. 2a-d, truncated triangular shaped monolayer $\mathrm{WS}_{2}$ crystals were obtained with $\mathrm{FWO}_{3}$ and $\mathrm{PTWO}_{3}$ precursors, while $\mathrm{WS}_{2}$ monolayers in a regular triangular shape could be achieved with $\mathrm{AWO}_{3}$ and $\mathrm{PTAWO}_{3}$ precursors, revealing the morphology modification caused by the various precursor pretreatment processes. The photoluminescence (PL) properties of $\mathrm{WS}_{2}$ monolayers in terms of the peak intensity and position can be dramatically changed due to the presence of various excitonic states such as trions, biexcitons and other charge bounded excitons, which strongly depend on the crystal quality and surface charge states of TMDC monolayers. ${ }^{26,44-47}$ As illustrated in Fig. 2e-l, the PL peak intensity mapping (Fig. 2e-h) and position mapping results (Fig. $2 \mathrm{i}-\mathrm{l}$ ) show that $\mathrm{WS}_{2}$ monolayers synthesized from the $\mathrm{AWO}_{3}$ precursor exhibit a more uniform PL peak intensity across the surface and have dominant PL peak with higher photon energy $(\sim 2.0 \mathrm{eV})$, which is closer to the band gap of $\mathrm{WS}_{2}(2.1 \mathrm{eV}),{ }^{48}$ as compared with the other three synthesized samples.

To evaluate the oxygen vacancies in the precursors, we characterized the precursors with X-ray powder diffraction (XRD) as shown in Fig. 3, S5 and S6. $\dagger$ XRD patterns of all the four precursors consist of the monoclinic phase of $\mathrm{WO}_{3}$ diffraction peaks (see Fig. 3a), showing that the precursor treatment process did not change the crystal phase. However, the enlarged view of the peak ranging from 22 to $26^{\circ}$ shows that all the precursors exhibit a peak position shift to a higher diffraction angle as compared to the standard XRD patterns for the monoclinic phase of $\mathrm{WO}_{3}$ (Fig. 3b). The XRD diffraction peaks from $\mathrm{AWO}_{3}$ powder exhibit the most significant peak shift $\left(0.1^{\circ}\right)$ to a higher diffraction angle with peak intensity reduction
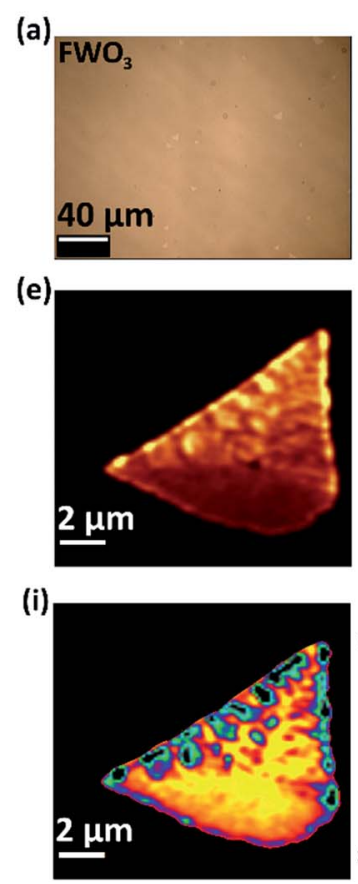

(b)

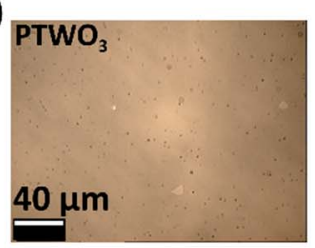

(f)
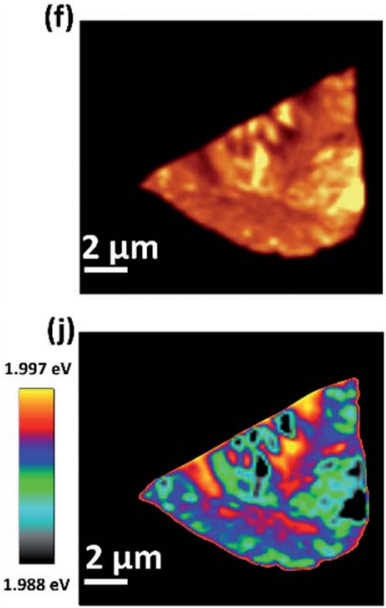

(c)

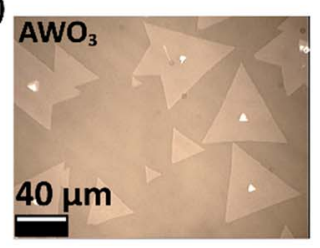

(g)
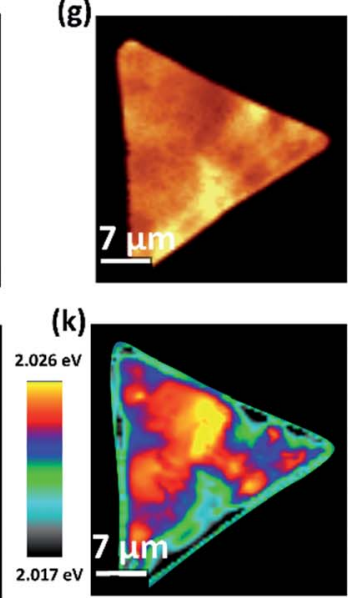

(d)

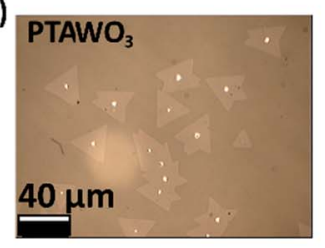

(h)
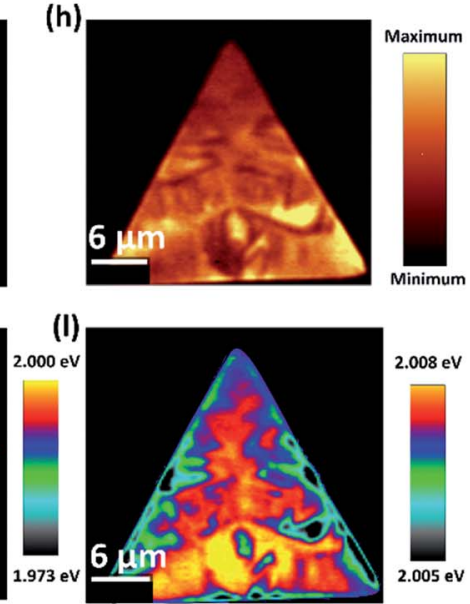

Fig. 2 Optical characterization of the monolayer $\mathrm{WS}_{2}$ crystals that were obtained from various types of pretreated $\mathrm{WO}_{3}$ powder. (a-d) Optical images of the monolayer $\mathrm{WS}_{2}$ crystals that were grown from $\mathrm{FWO}_{3}, \mathrm{PTWO}, \mathrm{AWO}_{3}$, and PTAWO $\mathrm{PT}_{3}$ powders, respectively. (e-h) PL intensity mapping images of the monolayer $\mathrm{WS}_{2}$ crystals that were synthesized from $\mathrm{FWO}_{3}, \mathrm{PTWO}_{3}, \mathrm{AWO}_{3}$ and $\mathrm{PTAWO} 3 \mathrm{Powders}$, respectively. (i-l) $\mathrm{PL}$ position mapping images of the monolayer $\mathrm{WS}_{2}$ crystals that were synthesized from $\mathrm{FWO}_{3}, \mathrm{PTWO}_{3}, \mathrm{AWO}_{3}$ and $\mathrm{PTAWO} 3$ powders, respectively. 
(a)

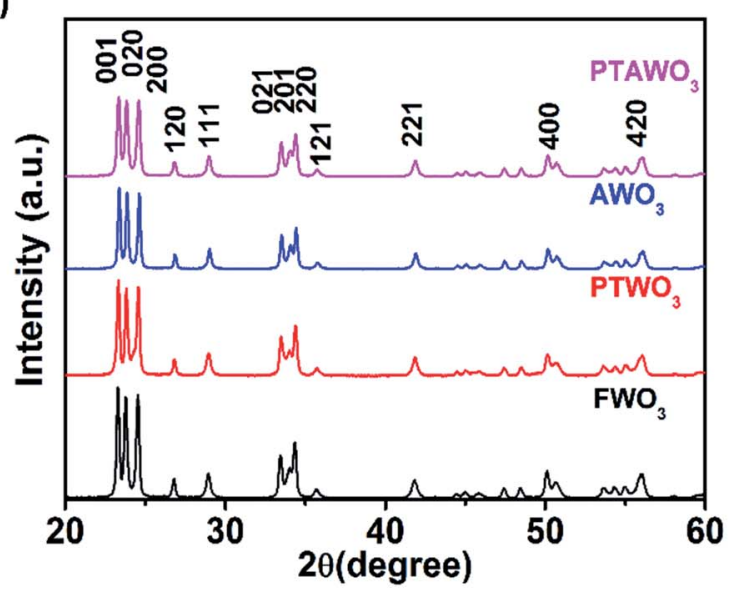

(b)

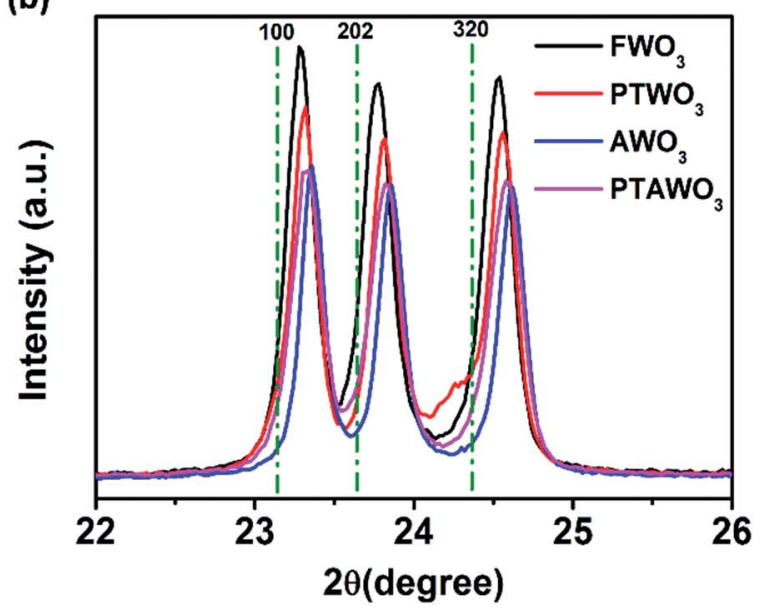

Fig. 3 (a) XRD patterns of $\mathrm{FWO}_{3}, \mathrm{PTWO}_{3}, \mathrm{AWO}_{3}$ and $\mathrm{PTAWO}_{3}$ powders. (b) Enlarged view of XRD patterns at 001,020 and 200 planes for $\mathrm{FWO}$, $\mathrm{PTWO}_{3}, \mathrm{AWO}_{3}$ and $\mathrm{PTAWO}$ powders.

as compared to $\mathrm{FWO}_{3}$, followed by $\mathrm{PTAWO}_{3}\left(0.07^{\circ}\right)$ and finally $\mathrm{PTWO}_{3}\left(0.03^{\circ}\right)$. The peak position shifts to a higher diffraction angle are related to the contraction of lattice parameters, which results from the formation of more oxygen vacancies and the degradation of the crystallinity of the $\mathrm{WO}_{3}$ powder due to the thermal annealing process. As shown in Fig. $\mathrm{S} 7, \dagger \mathrm{WO}_{3}$ powders prepared by different methods also show different colors from a greenish color to deep blue and these color changes have been attributed to the different oxygen vacancy contents in $\mathrm{WO}_{3}$ powders, which is in good consistency with the XRD results. ${ }^{30,49-51}$ To eliminate the effects of water content in the precursors on the growth of monolayer $\mathrm{WS}_{2}$, thermal gravimetric analysis (TGA) (see Fig. S8 $\dagger$ ) has been done and the results reveal that surface-absorbed water in the $\mathrm{WO}_{3}$ powders is less than $1 \%$, implying negligible effects on the growth of monolayer $\mathrm{WS}_{2}$.

To further confirm the oxygen vacancy induced peak position and intensity change in XRD spectra, we further examined the optimized crystal structure of $\mathrm{WO}_{3}$ with and without oxygen vacancies by theoretical calculations (see Table 1). The lattice structures of $\mathrm{WO}_{3}$ have been studied using density functional theory (DFT) calculations with plane-wave pseudopotentials. ${ }^{52}$ The initial space group of stoichiometric $\mathrm{WO}_{3}$ is $\mathrm{P}_{21} / \mathrm{a}(\# 14)$, belonging to the monoclinic space group. After structural optimization (Fig. 4), the system's space group converts to $\mathrm{Pbcm}$ (\#57). The optimized lattice parameters are $a=3.904 \AA, b=$ $7.778 \AA$, and $c=7.558 \AA$. The unit cell volume is $229.54 \AA^{3}$, and these four octahedrons in the system are equivalent. For the samples with oxygen vacancies, we remove one oxygen atom from the octahedron from three different directions, namely, $a$-, $b$-, and $c$-axis directions. For oxygen vacancies in the $a$-axis direction, the lattice parameter and volume decrease significantly. For oxygen vacancies in the $b$-axis direction, the bravais lattice shape changes, but the volume is substantially unchanged. For oxygen vacancies in the $c$-axis direction, the lattice constants of $a$ and $b$ decrease significantly, and the volume also decreases. In practice, oxygen vacancies may exist in any direction, and hence, from the above discussion, we can conclude that the presence of oxygen vacancies will cause the lattice constant of $\mathrm{WO}_{3}$ to shrink, which explains the peak position shift in XRD spectra for $\mathrm{WO}_{3}$ with different oxygen vacancy contents.

Moreover, X-ray photoelectron spectroscopy (XPS) analysis exhibits noticeable differences in the binding energy of tungsten and oxygen core levels as illustrated in Fig. 5, S9 and Table $\mathrm{S} 1 . \dagger$ The XPS peak fitting results from all $\mathrm{WO}_{3}$ powders show that besides the dominating $\mathrm{W} 4 \mathrm{f}^{(\mathrm{VI})}$ valence state, a portion of the $\mathrm{W} 4 \mathrm{f}^{(\mathrm{V})}$ valence state also exists, confirming the presence of oxygen vacancies in all the $\mathrm{WO}_{3}$ powders. Yet, the XPS spectral fitting of $\mathrm{W} 4 \mathrm{f}$ in $\mathrm{AWO}_{3}$ and $\mathrm{PTWO}_{3}$ powders suggests a higher contribution from the $\mathrm{W} 4 \mathrm{f}^{(\mathrm{V})}$ state as compared with that of $\mathrm{FWO}_{3}$ powder, indicating the higher oxygen vacancy concentration in both $\mathrm{AWO}_{3}$ and $\mathrm{PTWO}_{3}$ powders.

Table 1 Calculated optimized structural parameters of $\mathrm{WO}_{3}$ with and without oxygen vacancies by theoretical calculations

\begin{tabular}{|c|c|c|c|c|c|c|c|}
\hline \multirow[b]{2}{*}{ Crystal structure of $\mathrm{WO}_{3}$} & \multicolumn{3}{|c|}{ Lattice parameters $(\AA)$} & \multirow[b]{2}{*}{ Volume } & \multicolumn{3}{|c|}{ Angle (̊̊) } \\
\hline & $a$ & $b$ & $c$ & & $\alpha$ & $\beta$ & $\gamma$ \\
\hline Oxygen vacancies in the $a$-axis direction & 3.796 & 7.799 & 7.598 & 224.95 & 90.00 & 90.00 & 90.02 \\
\hline Oxygen vacancies in the $b$-axis direction & 3.808 & 7.795 & 7.573 & 229.48 & 90.00 & 90.00 & 90.03 \\
\hline Oxygen vacancies in the $c$-axis direction & 3.824 & 7.651 & 7.721 & 225.85 & 90.84 & 90.00 & 90.00 \\
\hline
\end{tabular}


(a)

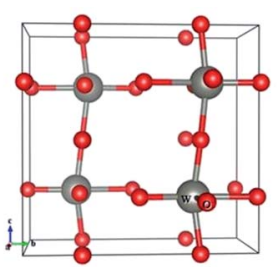

(c)

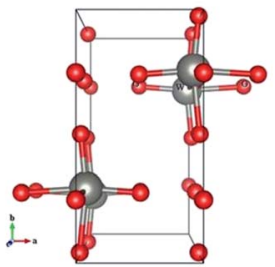

(b)

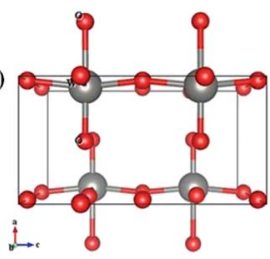

(d)

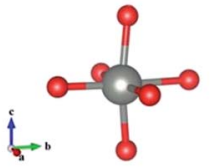

Fig. 4 The view of the optimized structure of $\mathrm{WO}_{3}$ from the (a) a-axis direction, (b) $b$-axis direction, and (c) $c$-axis direction and (d) the octahedron in the $\mathrm{WO}_{3}$ system. There are $4 \mathrm{~W}$ atoms and $12 \mathrm{O}$ atoms in this system.
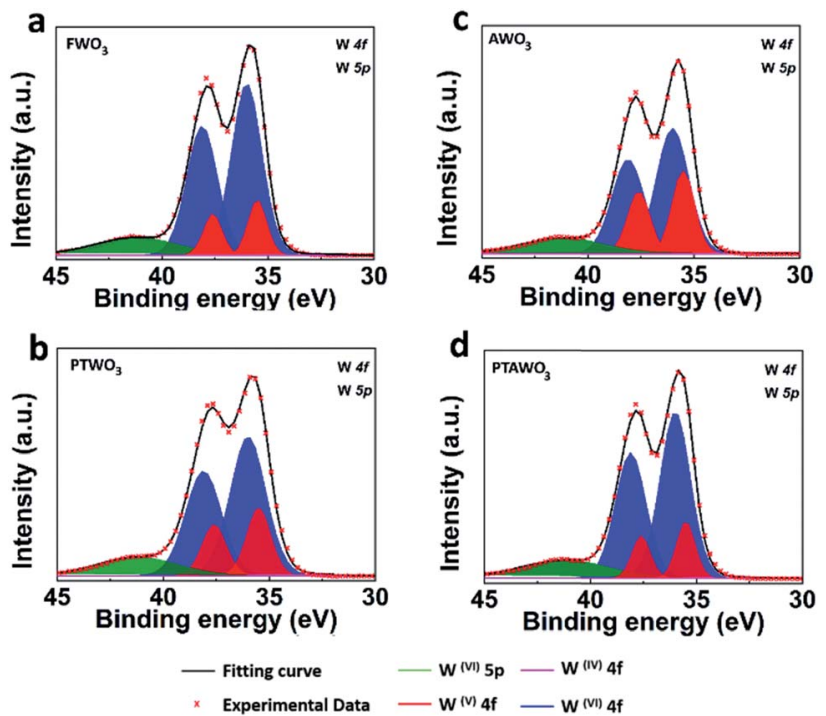

Fig. 5 XPS spectra fitting of $W 4 f$ and $W 5 p$ peaks for $W_{3}$ powders. (ad) XPS spectra of $\mathrm{W} 4 \mathrm{f}$ for $\mathrm{FWO}_{3}, \mathrm{PTWO}_{3}, \mathrm{AWO}_{3}$ and $\mathrm{PTAWO}_{3}$ powders, respectively.

Remarkably, compared with the other three control samples, a continuous dark oxide layer was formed after the plasma treatment of $\mathrm{WO}_{3}$ powder (Fig. S7b $\dagger$ ). As reported by Wang et al., Raman spectroscopy is an effective way to characterize the crystallinity of $\mathrm{WO}_{3}$ powder. ${ }^{27}$ As illustrated in Fig. S10, $\dagger$ all the $\mathrm{WO}_{3}$ powders exhibit the two typical dominating Raman peaks of crystalline $\mathrm{WO}_{3}$ centered at $808 \mathrm{~cm}^{-1}$ and $715 \mathrm{~cm}^{-1}$, except the $\mathrm{PTWO}_{3}$ powder which shows a lower peak intensity and broader stretching mode peak at around $808 \mathrm{~cm}^{-1}$. The significant difference in Raman spectra suggests a strong plasma treatment effect on the surface crystallinity of $\mathrm{WO}_{3}$.

In fact, XPS analysis shows that the $\mathrm{W} 4 \mathrm{f}^{(\mathrm{V})}$ surface state is slightly reduced by thermal annealing for the $\mathrm{PTWO}_{3}$ samples, which could be due to the increased surface oxidation rate

towards linear kinetics after plasma treatment. ${ }^{53}$ Therefore, the $\mathrm{PTAWO}_{3}$ powder possesses more $\mathrm{W} 4 \mathrm{f}^{(\mathrm{VI})}$ surface state compared with $\mathrm{PTWO}_{3}$. It is also noticed that XPS analysis and Raman characterization mainly reflect the surface information of chemical composition; meanwhile XRD presents the crystallinity of bulk sample powders. As shown in Fig. 3b, the XRD diffraction peaks from $\mathrm{PTAWO}_{3}$ powder exhibit a peak shift to a higher diffraction angle and peak intensity reduction, suggesting more oxygen vacancies formed by the annealing process. Combining the XRD, theoretical calculation and XPS analysis, we reach a conclusion that both thermal annealing and plasma treatment lead to the oxygen vacancy formation, while plasma treatment mainly affects the surface chemical states and the thermal annealing process can significantly change the metal oxide crystallinity and result in a smaller lattice spacing. Obviously, combining the precursor characterization and growth results, large-size triangular $\mathrm{WS}_{2}$ monolayer flakes could be produced with a higher oxygen vacancy concentration in the $\mathrm{WO}_{3}$ precursor source, while small-size truncated $\mathrm{WS}_{2}$ monolayers were generally obtained when the number of oxygen vacancies in the $\mathrm{WO}_{3}$ precursor reduced. The obtained morphology of the as-growth $\mathrm{WS}_{2}$ is largely related to the reaction conditions, such as the precursor concentration ratio, substrates used, gas flow rate. In pretreated precursors, the highly volatile tungsten suboxide will result in an increase of the amount of tungsten precursors, increase the rate of reaction toward sulfur and hence promote the growth of $\mathrm{WS}_{2}$. Overall, the thermal annealing process generates more oxygen vacancies in the $\mathrm{WO}_{3}$ precursor, which effectively promotes the growth of $\mathrm{WS}_{2}$ monolayers with larger size and better crystallinity. Besides, the PL results also suggest that the monolayer $\mathrm{WS}_{2}$ samples synthesized from thermal annealing pretreated precursors may have a lower lattice structure defect concentration.

To better reveal the superiority of the monolayer $\mathrm{WS}_{2}$ obtained from the $\mathrm{AWO}_{3}$ powder, we further explored the effect of the annealing atmosphere on the structure of the $\mathrm{WS}_{2}$. As aforementioned above, in previous studies, hydrogen gas $\left(\mathrm{H}_{2}\right)$ was introduced to promotes the reduction of $\mathrm{WO}_{3}$ and hence promote the growth of $\mathrm{WS}_{2}$ monolayers. ${ }^{\mathbf{1 6 , 1 9 , 2 6 , 4 4}}$ However, an excessive hydrogen supply could also induce defects. Therefore, we further study the quality of $\mathrm{WS}_{2}$ monolayers synthesized with a thermally annealed precursor. The thermally annealed precursor synthesized $\mathrm{WS}_{2}\left(\mathrm{AWS}_{2}\right)$ sample shows a uniform PL intensity feature on the whole surface and still retains its optical properties after being transferred onto a silicon dioxide substrate or more than one-month after the synthesis (see Fig. S11 and S12 $\dagger$ ). There is no aggregation/restacking effect observed for the $\mathrm{AWS}_{2}$ monolayers for prolonged periods (after one month). The optical image and optical characterization such as Raman and PL characterization (see Fig. S12†) show the good stability of the as-grown monolayer $\mathrm{AWS}_{2}$ after being exposed to ambient air for more than one month. The XPS spectra of W4f and S2p peak in $\mathrm{AWS}_{2}$ as shown in Fig. $\mathrm{S} 13 \dagger$ confirm the presence of $\mathrm{WS}_{2}$.

Besides, atomic resolution annular dark field scanning transmission electron microscope (ADF-STEM) Z-contrast 

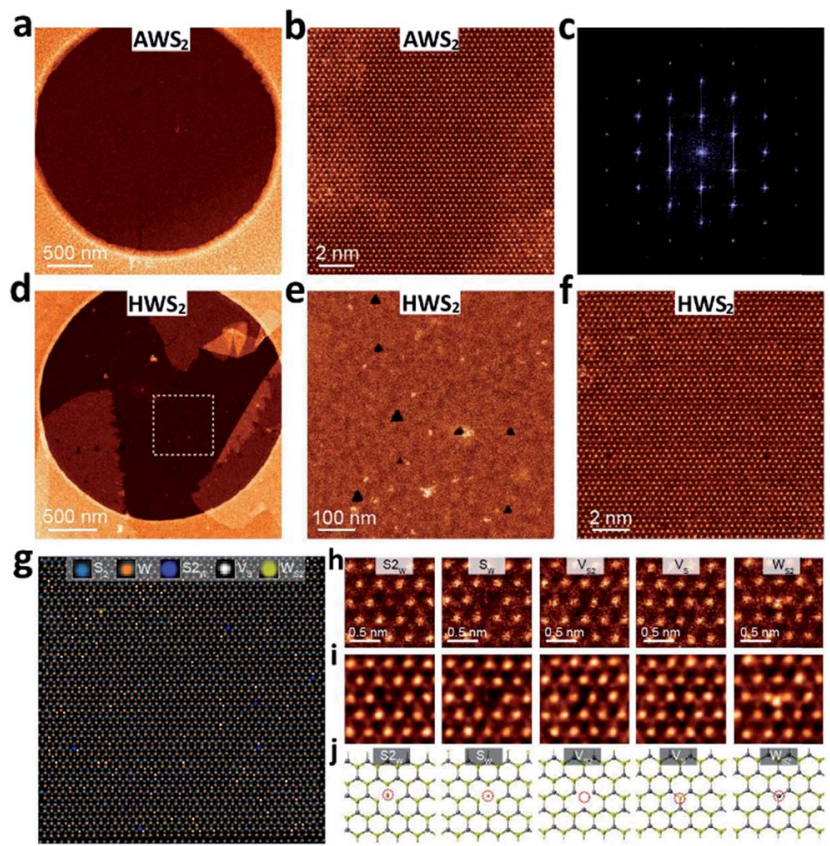

Fig. 6 Atomic structure of monolayer $\mathrm{WS}_{2}$. (a) STEMADF image of the as-transferred $\mathrm{AWS}_{2}$ monolayer onto a Quantifoil grid. (b) Atomic resolution ADF/STEM image of the AWS 2 crystal. (c) SAED patterns of the $\mathrm{WS}_{2}$ crystal. (d) STEMADF image of the as-transferred $\mathrm{HWS}_{2}$ monolayer onto a Quantifoil grid. (e-f) Atomic resolution ADF/STEM image of the HWS 2 crystal. (g) Defect mapping of the HWS $\mathrm{HW}_{2}$ monolayer. (h) Atomic resolution ADF/STEM images of various intrinsic point defects present in monolayer $\mathrm{HWS}_{2}$, including monosulfur vacancies $\left(V_{s}\right)$, disulfur vacancies $\left(V_{S_{2}}\right)$, and antisite defects where a $W$ atom substituting a $\mathrm{S}_{2} / \mathrm{S}$ column $\left(\mathrm{W}_{\mathrm{S} 2}, \mathrm{Ws}\right)$ or a $\mathrm{S}_{2}$ column substituting a W atom $\left(\mathrm{S} 2_{\mathrm{w}}\right)$ is observed. (i) Processed atomic resolution ADF/STEM image of various intrinsic point defects present in monolayer $\mathrm{HWS}_{2}$. (j) Relaxed structural models of the experimentally observed point defects of $\mathrm{HWS}_{2}$.

images for the $\mathrm{AWS}_{2}$ and hydrogenated environmentally synthesized $\mathrm{WS}_{2}\left(\mathrm{HWS}_{2}\right)$ samples were also obtained to study the atomic defect level in both samples (see Fig. 6). The obtained ADF-STEM image and the defect mapping analysis (see Fig. $6 \mathrm{~b}$ and $\mathrm{S14} \dagger$ ) show that the $\mathrm{AWS}_{2}$ sample is almost defectfree, while there are a considerable number of lattice defects, including monosulfur vacancies $\left(V_{\mathrm{s}}\right)$, disulfur vacancies $\left(V_{\mathrm{S} 2}\right)$, and antisite defects where a $\mathrm{W}$ atom substituting a $\mathrm{S}_{2} / \mathrm{S}$ column $\left(\mathrm{W}_{\mathrm{S} 2}, \mathrm{~W}_{\mathrm{S}}\right)$ or a $\mathrm{S}_{2}$ column substituting a $\mathrm{W}$ atom $\left(\mathrm{S}_{\mathrm{w}}\right)$ is observed in the $\mathrm{HWS}_{2}$ sample (see Fig. $6 \mathrm{~d}-\mathrm{j}$ ). These results suggest that the $\mathrm{HWS}_{2}$ sample contains more lattice defects than the $\mathrm{AWS}_{2}$ sample. This clearly illustrated that the $\mathrm{AWS}_{2}$ exhibits better crystallinity and fewer structural defects.

\section{Conclusions}

In conclusion, we have comprehensively studied the effects of various stoichiometric $\mathrm{WO}_{3}$ precursors on the growth of $\mathrm{WS}_{2}$ monolayers. The morphology of $\mathrm{WS}_{2}$ monolayers transforms from a truncated triangle to a triangular shape when the oxygen vacancies in the $\mathrm{WO}_{3}$ precursor increase, resulting in the change of PL properties and crystallinity in the as-growth $\mathrm{WS}_{2}$ monolayers. The number of oxygen vacancies in $\mathrm{WO}_{3}$ induces a change in the growth atmosphere and hence significantly affects the lateral growth morphology as well as the optical properties of monolayer $\mathrm{WS}_{2}$. In our study, the annealed $\mathrm{WO}_{3}$ precursor with the highest oxygen vacancy concentration generates monolayer $\mathrm{WS}_{2}$ crystals with improved quality in terms of lateral size, surface coverage, and crystallinity, while $\mathrm{PTWO}_{3}$ and $\mathrm{AWO}_{3}$ powders with a lower oxygen vacancy concentration suppress the growth of monolayer $\mathrm{WS}_{2}$. Our results demonstrate the possibility of tailoring the morphology and crystal quality of $\mathrm{WS}_{2}$ monolayers by the tuning of oxygen vacancy content in the metal oxide precursor. The investigation on various non-stoichiometric $\mathrm{WO}_{3}$ precursors provides better understanding on the growth mechanism of large-area, highquality $\mathrm{WS}_{2}$ monolayers.

\section{Conflicts of interest}

There are no conflicts to declare.

\section{Acknowledgements}

This work is supported by A*STAR AME IRG (Grant no. A1783c0011). Y. S. acknowledges the support from the Science and Technology Project of Shenzhen (JCYJ20170817101100705), the Thousand Young Talents Program of China, the National Natural Science Foundation of China (Grant no. 51602200) and the (Key) Project of Department of Educational Commission of Guangdong Province (Grant no. 2016KZDXM008). This project was supported by the Shenzhen Peacock Plan (Grant no. KQTD2016053112042971).

\section{References}

1 Z. Sun, A. Martinez and F. Wang, Nat. Photonics, 2016, 10, 227-238.

2 G. Grosso, J. Graves, A. T. Hammack, A. A. High, L. V. Butov, M. Hanson and A. C. Gossard, Nat. Photonics, 2009, 3, 577580.

3 C. Gong, H. Zhang, W. Wang, L. Colombo, R. M. Wallace and K. Cho, Appl. Phys. Lett., 2015, 107, 139904.

4 H. Tan, Y. Fan, Y. Zhou, Q. Chen, W. Xu and J. H. Warner, ACS Nano, 2016, 10, 7866-7873.

5 A. P. Nayak, Z. Yuan, B. Cao, J. Liu, J. Wu, S. T. Moran, T. Li, D. Akinwande, C. Jin and J. F. Lin, ACS Nano, 2015, 9, 91179123.

6 T. Chu, H. Ilatikhameneh, G. Klimeck, R. Rahman and Z. Chen, Nano Lett., 2015, 15, 8000-8007.

7 W. X. Zhang, Z. S. Huang, W. L. Zhang and Y. R. Li, Nano Res., 2014, 7, 1731-1737.

8 K. F. Mak, C. Lee, J. Hone, J. Shan and T. F. Heinz, Phys. Rev. Lett., 2010, 105, 136805.

9 W. Zhao, Z. Ghorannevis, L. Chu, M. Toh, C. Kloc, P. H. Tan and G. Eda, ACS Nano, 2013, 7, 791-797.

10 H. Shi, H. Pan, Y. W. Zhang and B. I. Yakobson, Phys. Rev. B: Condens. Matter Mater. Phys., 2013, 87, 155304. 
11 X. Liu, J. Hu, C. Yue, N. Della Fera, Y. Ling, Z. Mao and J. Wei, ACS Nano, 2014, 8, 10396-10402.

12 M. W. Iqbal, M. Z. Iqbal, M. F. Khan, M. A. Shehzad, Y. Seo, J. H. Park, C. Hwang and J. Eom, Sci. Rep., 2015, 5, 10699.

13 L. Su, Y. Yu, L. Cao and Y. Zhang, Nano Res., 2015, 8, 26862697.

14 R. K. Ghosh and S. Mahapatra, IEEE J. Electron Devices Soc., 2013, 1, 175-180.

15 H. R. Gutiérrez, N. Perea-López, A. L. Elías, A. Berkdemir, B. Wang, R. Lv, F. López-Urías, V. H. Crespi, H. Terrones and M. Terrones, Nano Lett., 2013, 13, 3447-3454.

16 K. M. McCreary, A. T. Hanbicki, G. G. Jernigan, J. C. Culbertson and B. T. Jonker, Sci. Rep., 2016, 6, 19159.

17 E. J. Sie, J. W. McIver, Y. H. Lee, L. Fu, J. Kong and N. Gedik, Nat. Mater., 2015, 14, 290-294.

18 H. Zeng, G. B. Liu, J. Dai, Y. Yan, B. Zhu, R. He, L. Xie, S. Xu, X. Chen, W. Yao and X. Cui, Sci. Rep., 2013, 3, 1608.

19 H. Li, Y. Li, A. Aljarb, Y. Shi and L. J. Li, Chem. Rev., 2018, 118, 6134-6150.

20 Z. Cai, B. Liu, X. Zou and H. M. Cheng, Chem. Rev., 2018, 118, 6091-6133.

21 J. Chen, X. Zhao, S. J. R. Tan, H. Xu, B. Wu, B. Liu, D. Fu, W. Fu, D. Geng, Y. Liu, W. Liu, W. Tang, L. Li, W. Zhou, T. C. Sum and K. P. Loh, J. Am. Chem. Soc., 2017, 139, 1073-1076.

22 Y. Shi, H. Li and L. J. Li, Chem. Soc. Rev., 2015, 44, 2744-2756.

23 S. L. Wong, H. Liu and D. Chi, Prog. Cryst. Growth Charact., 2016, 62, 9-28.

24 Y. Sheng, H. Tan, X. Wang and J. H. Warner, Chem. Mater., 2017, 29, 4904-4911.

25 S. H. Choi, S. Boandoh, Y. H. Lee, J. S. Lee, J. H. Park, S. M. Kim, W. Yang and K. K. Kim, ACS Appl. Mater. Interfaces, 2017, 9, 43021-43029.

26 K. N. Kang, K. Godin and E.-H. Yang, Sci. Rep., 2015, 5, 13205.

27 X. Zhao, J. Feng, S. Chen, Y. Huang, T. C. Sum and Z. Chen, Phys. Chem. Chem. Phys., 2017, 19, 1074-1082.

28 Z. Lou and C. Xue, CrystEngComm, 2016, 18, 8406-8410.

29 G. Wang, Y. Yang, Y. Ling, H. Wang, X. Lu, Y. C. Pu, J. Z. Zhang, Y. Tong and Y. Li, J. Mater. Chem. A, 2016, 4, 2849-2855.

30 R. Chatten, A. V. Chadwick, A. Rougier and P. J. D. Lindan, J. Phys. Chem. B, 2005, 109, 3146-3156.

31 W. Wang, A. Janotti and C. G. Van de Walle, J. Mater. Chem. C, 2016, 4, 6641-6648.

32 J. D. Cain, F. Shi, J. Wu and V. P. Dravid, ACS Nano, 2016, 10, 5440-5445.
33 Y. Shi, C. Hamsen, X. Jia, K. K. Kim, A. Reina, M. Hofmann, A. L. Hsu, K. Zhang, H. Li, Z. Y. Juang, M. S. Dresselhaus, L. J. Li and J. Kong, Nano Lett., 2010, 10, 4134-4139.

34 Y. Shi, H. Li, J. I. Wong, X. Zhang, Y. Wang, H. Song and H. Y. Yang, Sci. Rep., 2015, 5, 10378.

35 J. P. Perdew, K. Burke and M. Ernzerhof, Phys. Rev. B: Condens. Matter Mater. Phys., 1996, 77, 3865-3868.

36 G. Kresse and D. Joubert, Phys. Rev. B: Condens. Matter Mater. Phys., 1999, 59, 1758-1775.

37 G. Kresse and J. Furthmüller, Phys. Rev. B: Condens. Matter Mater. Phys., 1996, 54, 11169-11186.

38 H. J. Monkhorst and J. D. Pack, Phys. Rev. B: Solid State, 1976, 13, 5188-5192.

39 M. G. Charlton, Nature, 1954, 174, 703.

40 N. Birks, G. H. Meier and F. S. Pettit, Introduction to the High Temperature Oxidation of Metals, Cambridge University Press, Cambridge, 2 edn, 2006.

41 V. K. Sarin, J. Mater. Sci., 1975, 10, 593-598.

42 P. K. Sahoo, S. Memaran, Y. Xin, L. Balicas and H. R. Gutiérrez, Nature, 2018, 553, 63.

43 M. S. Kim, S. J. Yun, Y. Lee, C. Seo, G. H. Han, K. K. Kim, Y. H. Lee and J. Kim, ACS Nano, 2016, 10, 2399-2405.

44 S. Paradisanos, N. T. Germanis, C. Pelekanos, E. Fotakis, E. Kymakis, G. Kioseoglou and E. Stratakis, Appl. Phys. Lett., 2017, 110, 193102.

45 Y. C. Lin, S. Li, H. P. Komsa, L. J. Chang, A. V. Krasheninnikov, G. Eda and K. Suenaga, Adv. Funct. Mater., 2018, 28, 1704210.

46 J. H. Yun, J. Youngjo, Y. S. Joon, Z. Jiong, B. Jaeyoon, K. D. Hoon, L. H. Seok and L. Y. Hee, Adv. Mater., 2017, 29, 1605043.

47 G. Plechinger, P. Nagler, J. Kraus, N. Paradiso, C. Strunk, C. Schüller and T. Korn, Phys. Status Solidi RRL, 2015, 9, 457-461.

48 X. H. Wang, J. Q. Ning, Z. C. Su, C. C. Zheng, B. R. Zhu, L. Xie, H. S. Wu and S. J. Xu, RSC Adv., 2016, 6, 27677-27681.

49 C. G. Granqvist, Handbook of Inorganic Electrochromic Materials, Elsevier, 1995.

50 G. Wang, Y. Ling, H. Wang, X. Yang, C. Wang, J. Z. Zhang and Y. Li, Energy Environ. Sci., 2012, 5, 6180-6187.

51 Y. Djaoued, S. Balaji and R. Brüning, J. Nanomater., 2012, 2012, 9.

52 J. Ihm, A. Zunger and M. L. Cohen, J. Phys. C: Solid State Phys., 1979, 12, 4409.

53 S. C. Cifuentes, M. A. Monge and P. Pérez, Corros. Sci., 2012, 57, 114-121. 